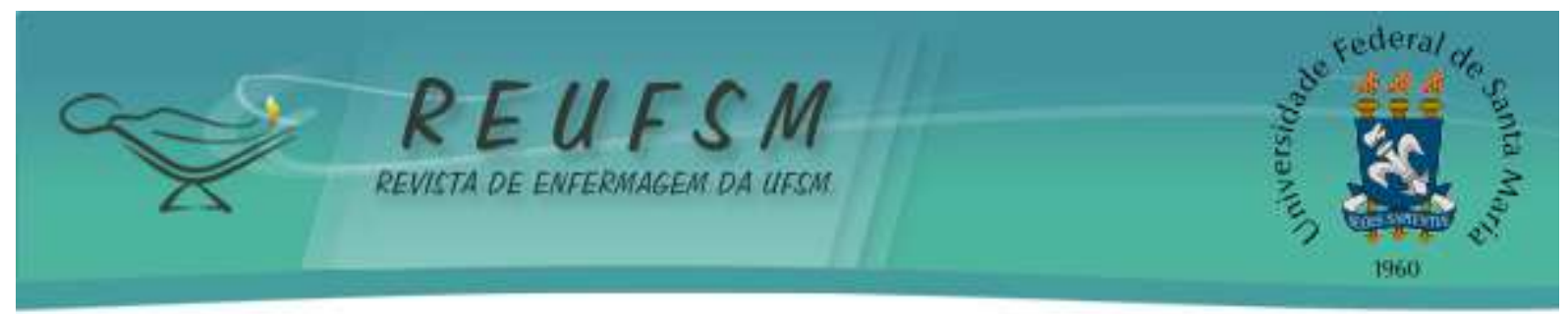

\title{
PERCEPÇÃO DA ENFERMAGEM SOBRE A QUALIDADE DO ACOLHIMENTO COM CLASSIFICAÇÃO DE RISCO DO SERVIÇO DE EMERGÊNCIA
}

\section{NURSING PERCEPTION OF THE QUALITY OF EMERGENCY TRIAGE \\ PERCEPCIÓN DE LA ENFERMERÍA SOBRE LA CALIDAD DE LA ACOGIDA CON CLASIFICACIÓN DE RIESGO DEL SERVICIO DE EMERGENCIA}

\author{
Thamy Caamaño Droguett ${ }^{1}$ \\ Meiry Fernanda Pinto Okuno ${ }^{2}$ \\ Cassia Regina Vancini Campanharo ${ }^{3}$ \\ Maria Carolina Barbosa Teixeira Lopes ${ }^{4}$ \\ Ruth Ester Assayag Batista ${ }^{5}$
}

\section{Doi: $10.5902 / 2179769228748$}

RESUMO: Objetivo: avaliar a qualidade do Acolhimento com Avaliação e Classificação de Risco de um serviço de emergência segundo percepção dos profissionais de enfermagem. Método: estudo transversal realizado no Acolhimento com Avaliação e Classificação de Risco do serviço de emergência do Hospital São Paulo, entre março e julho de 2016. Participaram 133 profissionais que atuavam no setor no mínimo há três meses. Foi utilizado questionário com variáveis para caracterização sociodemográfica e instrumento para Avaliação do Acolhimento com Classificação de Risco. Resultados: idade média 36,2 anos, maioria do sexo feminino $(77,4 \%)$ e formado há mais de 5 anos $(59,1 \%)$. O Acolhimento com Avaliação e Classificação de Risco obteve as seguintes pontuações: estrutura $(23,0)$, processo $(22,0)$ e resultado $(20,9)$. Conclusão: o Acolhimento com Avaliação e Classificação de Risco foi avaliado como precário na percepção dos profissionais de enfermagem.

Descritores: Acolhimento; Serviços médicos de emergência; Enfermagem em emergência; Classificação; Risco

ABSTRACT: Aim: to evaluate the quality of triage in an emergency service according to the perception of the nursing professionals. Method: a cross-sectional study carried out at the emergency service triage of the São Paulo Hospital, between March and July 2016. A hundred and thirty-three professionals who worked in the sector for at least three months participated in the study. A questionnaire was used with variables for sociodemographic characterization and, also, an instrument for triage quality evaluation. Results: mean age 36.2 years, the majority of females $(77.4 \%)$ and graduated for more than 5 years $(59.1 \%)$. The triage obtained the following scores: structure (23.0), process (22.0) and result (20.9). Conclusion: the triage quality was evaluated as precarious in the perception of the nursing professionals.

Descriptors: Triage; Emergency medical services; Emergency nursing; Quality of Health Care

\footnotetext{
${ }^{1}$ Enfermeira, Especialista em Urgência e Emergência, Hospital Brigadeiro. São Paulo, SP, Brasil. E-mail: thaminha@gmail.com

${ }^{2}$ Enfermeira, PhD, Escola Paulista de Enfermagem, Universidade Federal de São Paulo. São Paulo, SP, Brasil. Email: mf.pinto@unifesp.br

${ }^{3}$ Enfermeira, PhD, Escola Paulista de Enfermagem, Universidade Federal de São Paulo. São Paulo, SP, Brasil. Email: cvancini@unifesp.br

${ }^{4}$ Enfermeira, MSc, Escola Paulista de Enfermagem, Universidade Federal de São Paulo. São Paulo, SP, Brasil. E-mail: lopes.carolina@unifesp.br

${ }^{5}$ Enfermeira, Pós-doutor, Escola Paulista de Enfermagem, Universidade Federal de São Paulo. São Paulo, SP, Brasil. E-mail: ruth.ester@unifesp.br
} 


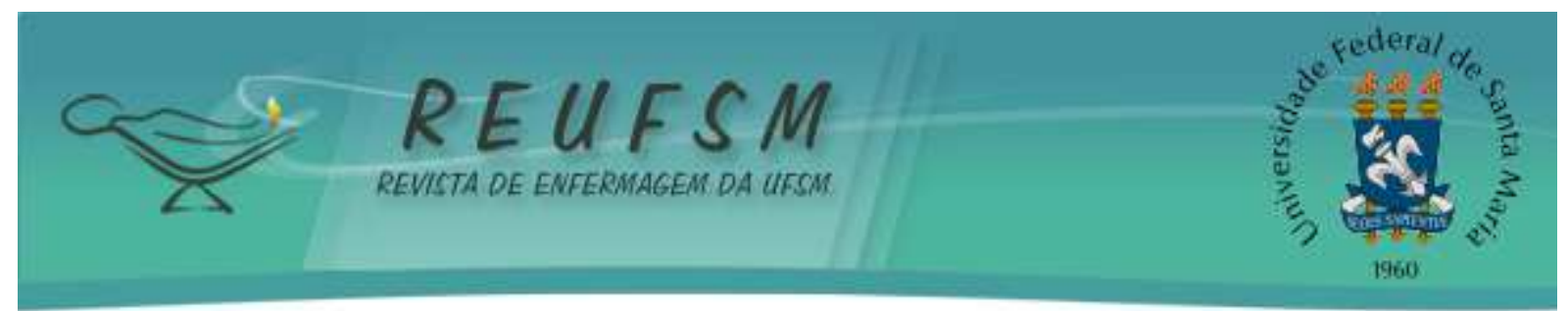

RESUMEN: Objetivo: evaluar la calidad de la Acogida con Evaluación y Clasificación de Riesgo de un servicio de emergencia a partir de la percepción de los profesionales de la enfermería. Método: estudio transversal, realizado en la Acogida con Evaluación y Clasificación de Riesgo, del servicio de emergencia del Hospital São Paulo, entre marzo y julio de 2016. Participaron 133 profesionales que actuaban en ese sector por pelo menos tres meses. Utilización de cuestionario con variables para caracterización sociodemográfica e instrumento para Evaluación de Acogida con Clasificación de Riesgo. Resultados: edad media 36,2 años, mayoría del sexo femenino (77,4\%) y con grado académico por más de 5 años (59,1\%). La Acogida con Evaluación y Clasificación de Riesgo obtuvo puntuaciones: estructura $(23,0)$, proceso (22,0) y resultado (20,9). Conclusión: la Acogida con Evaluación y Clasificación de Riesgo fue evaluada como precaria en la percepción de los profesionales de enfermería.

Descriptores: Triaje; Servicios médicos de emergencia; Enfermería de emergencia, Calidad de la atención de salud

\section{INTRODUÇÃO}

A superlotação é um fenômeno da saturação do Serviço de Emergência (SE) e é definido como o desequilíbrio simultâneo entre a procura por cuidados de saúde e a capacidade de resposta do sistema. É uma consequência da escassez de leitos disponíveis, tanto a nível hospitalar como no próprio SE. Representa um problema que afeta a maioria dos países, independentemente de sua condição socioeconômica, tornando-se um problema de gestão de todo o hospital e não exclusivo do SE e, por suas implicações, tornou-se um problema de saúde pública cada vez mais importante. Superlotação do SE dificulta o cuidado adequado ao paciente principalmente quando o tempo de início do tratamento é essencial, provoca atrasos no diagnóstico e, consequentemente, no início do tratamento, está associado com o aumento da morbidade e mortalidade, favorece o erro humano, e aumenta a permanência hospitalar, bem como os custos. ${ }^{1}$

Uma das estratégias adotadas para organizar o atendimento nos SE é o Acolhimento com Avaliação e Classificação de Risco (AACR). Em diversos países, como Inglaterra, Estados Unidos, e Canadá, os enfermeiros utilizam fluxogramas para priorização do atendimento, de acordo com as necessidades apresentadas pelos usuários, direcionando-os à assistência adequada, com a finalidade de reduzir o tempo de espera. No Brasil, o desempenho dessa atividade pelo enfermeiro é relativamente novo. ${ }^{2}$

O AACR é uma ferramenta que, além de garantir atendimento imediato do usuário com grau de risco elevado, propicia informações aos usuários sobre sua condição de saúde e o tempo de espera; promove o trabalho em equipe; melhora as condições de trabalho dos profissionais de 


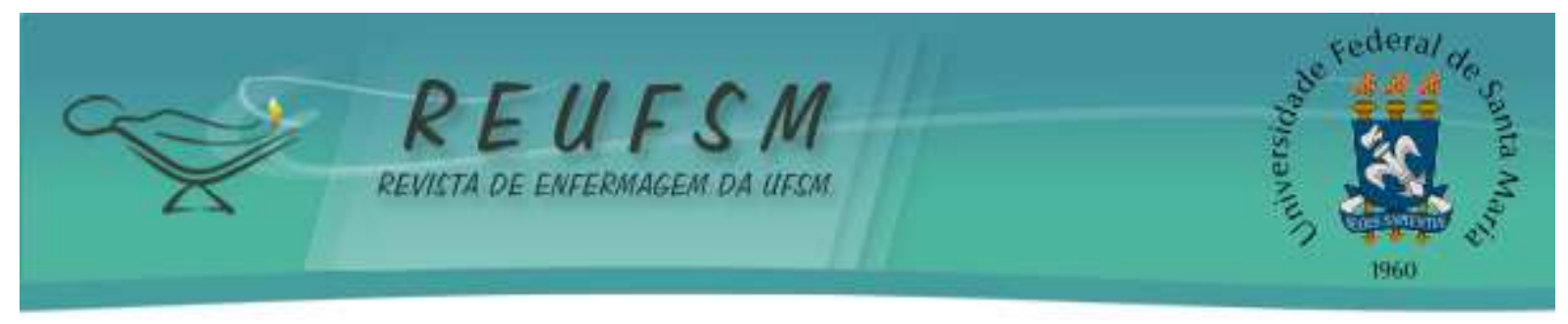

saúde por meio de discussão da ambiência e implantação do cuidado horizontalizado; aumenta a satisfação dos usuários e fomenta a pactuação entre os serviços da rede assistencial. ${ }^{2}$

Os protocolos assistenciais sistematizam a ação do profissional, além de serem fundamentais para a efetiva AACR e avaliação da vulnerabilidade do paciente. A avaliação dos enfermeiros que utilizaram o protocolo, pode refletir a necessidade de uma tecnologia em saúde consistente e eficaz para uma classificação de risco segura, além de apontar para algumas adaptações necessárias instituido um protocolo de acordo com a necessidade dos profissionais e a realidade do serviço. ${ }^{3}$

A criação de sistemas de qualidade em saúde deve ser uma ação prioritária dos serviços se saúde e os enfermeiros, necessitam assumir um papel fundamental na definição de padrões de qualidade dos cuidados prestados. A enfermagem é o maior grupo profissional dentro das organizações de saúde, onde a comunidade, espera que os cuidados prestados sejam de qualidade para diagnosticar e cuidar saúde e doença, para que o paciente seja independente e tenha uma vida mais saudável. ${ }^{4}$

Portanto, a qualidade dos serviços de saúde vem sendo discutida nas organizações envolvidas, com o objetivo desenvolver programas e ações capazes de atender as necessidades e as expectativas dos usuários. Uma forma de medir a qualidade dos serviços de saúde é por meio da utilização de indicadores, sendo considerada uma unidade de medida de um evento ou atividade, e empregado como um guia para monitorar e avaliar a qualidade da assistência aos clientes ou às atividades de um serviço. ${ }^{5} \mathrm{O}$ AACR ao distinguir os pacientes mais graves dos menos graves e oferecer atendimento de acordo com o grau de prioridade, pode ser utilizado como um indicador para mensurar a qualidade da assistência em serviço de emergência. ${ }^{2,5}$

Quando se pensa em qualidade nos serviços hospitalares, surge a necessidade de discutir estratégias de gestão que dêem suporte às instituições, para que possam atender às necessidades e exigências do paciente em todas as suas dimensões. ${ }^{6}$

No Brasil, a partir da década de 1930, acentuaram-se debates a respeito da melhoria na qualidade dos serviços hospitalares. Desde então, a criação de fichas, protocolos, sistemas, programas e políticas têm ganhado espaço nas discussões sobre planejamento da gestão hospitalar. ${ }^{6}$

Dentre todos os setores de um hospital, é provável que o SE seja um dos mais complexos para a implantação de sistemas que visam à melhoria da qualidade, porque as dificuldades observadas nesse local são distintas dos outros setores hospitalares, pelo fato de, 


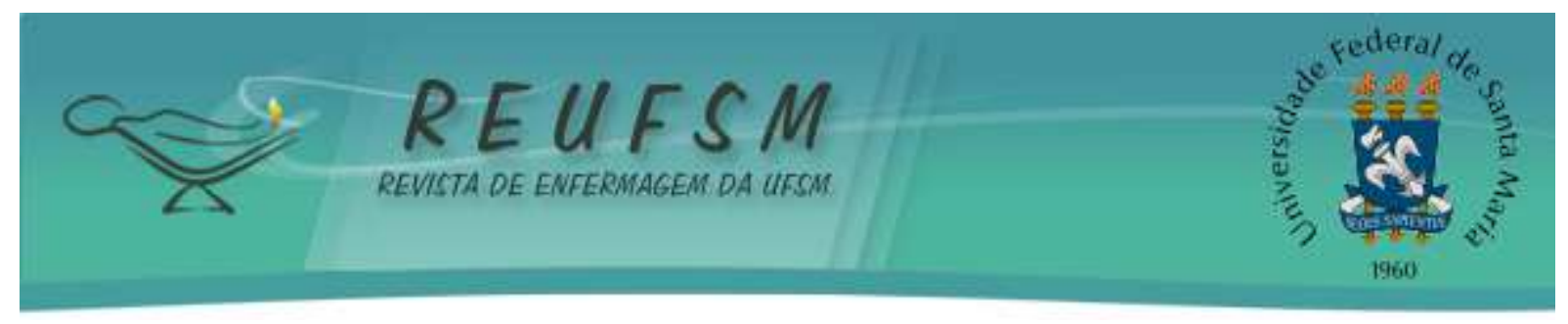

cotidianamente, estar superlotado; atuar sobre processos de trabalho fragmentados; apresentar conflitos e assimetria de poder, dentre outros. ${ }^{2}$

Sabe-se que, de modo geral, os SE de outros países também apresentam dificuldades no atendimento devido à alta demanda. ${ }^{5}$ No Brasil não é diferente, mas a utilização do ACCR parece minimizar os seus efeitos, pois estudos acerca do tema apontam que essa diretriz tem produzido melhorias no fluxo de atendimento, mesmo em instituições cuja adesão ocorreu em situações consideradas de difícil solução. ${ }^{5}$

Devido às melhorias que o ACCR tem proporcionado nos SE, considera-se que essa diretriz seja uma das principais ferramentas de apoio à obtenção da qualidade no atendimento "de porta" dos serviços de emergências dos hospitais brasileiros. ${ }^{5}$

Diante do exposto, a monitorização dos processos dentro das instituições tem sido muito valorizada, devido à possibilidade de implantação de estratégias que possam evitar a ocorrência de erros. Neste contexto, a avaliação da qualidade do AACR pode melhorar a segurança e a qualidade da assistência à saúde aos pacientes no SE.

Desse modo, para responder a questão: qual a percepção da equipe de enfermagem acerca da qualidade do AACR, realizou-se este estudo teve com o objetivo avaliar a qualidade do Acolhimento com Avaliação e Classificação de Risco de um serviço de emergência segundo percepção dos profissionais de enfermagem.

\section{MÉTODO}

Estudo transversal e descritivo de abordagem quantitativa, realizado no setor de Acolhimento com Avaliação e Classificação de Risco Serviço Emergência, localizado no Hospital São Paulo (HSP). Os participantes do estudo assinaram um Termo de Consentimento Livre Esclarecido, assegurado o anonimato das participantes, segundo as normas da Resolução no 466/12 do Conselho Nacional de Saúde do Ministério da Saúde.

O AACR funciona 24 horas por dia, sete dias por semana, sendo realizado por enfermeiro. Durante a entrevista, o paciente é questionado sobre a queixa, início do quadro, antecedentes pessoais, medicações em uso e alergias. Os sinais vitais são aferidos e é atribuída uma cor ao paciente para cada caso, o mesmo encaminhado para as especialidades clínicas (clínica médica, neurologia e psiquiatria) ou cirúrgicas (cirurgia geral, ginecologia, neurocirurgia, otorrinolaringologia e ortopedia). Ressalta-se que especialidades de pediatria e 


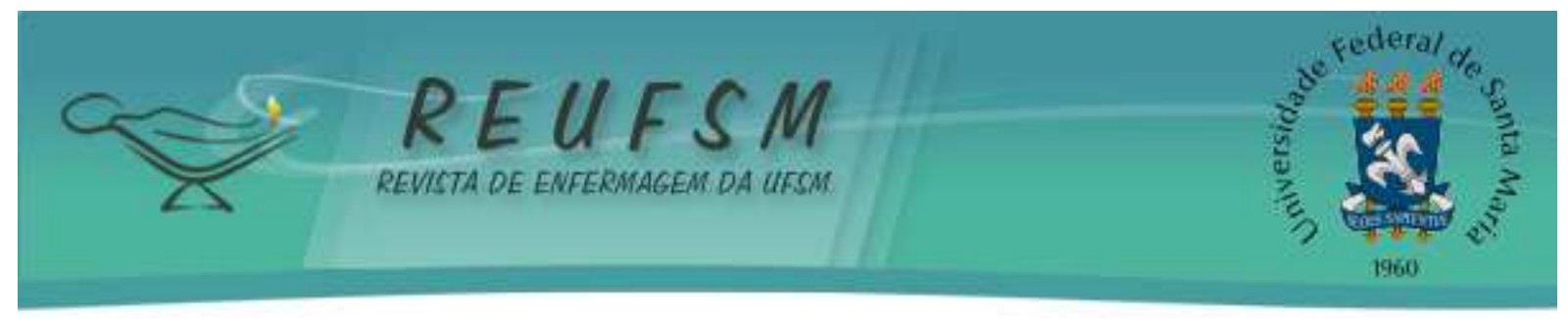

oftalmologia têm classificação realizada por médicos. Informações são registradas na ficha de atendimento e armazenadas no sistema de informação institucional.

O protocolo de AACR utilizado no Hospital São Paulo é institucional, com base no protocolo do Ministério da Saúde, porém utiliza cinco níveis de cores semelhante ao Protocolo de Manchester. ${ }^{7-8} \mathrm{O}$ enfermeiro é o profissional que executa esta atividade, classificando o paciente por cores de acordo com a gravidade. As cores utilizadas e os tempos preconizados são respectivamente: vermelha (atendimento imediato), laranja (atendimento em até 10 minutos), amarela (atendimento em até 60 minutos), verde (atendimento em até 120 minutos) e azul (atendimento em até 240 minutos).

O estudo foi realizado entre março de 2016 e julho de 2016. A amostra foi de conveniência, ou seja, foram incluídos os profissionais que estavam no plantão de trabalho, no período em que a pesquisadora estava no Serviço de Emergência realizando a coleta de dados.

A pesquisadora explicou os objetivos do estudo e a coleta de dados ocorreu após leitura e assinatura do termo de consentimento livre e esclarecido pelos participantes da pesquisa. A entrevista foi realizada pela mesma pesquisadora com duração média de 30 minutos.

Para obtenção dos dados sociodemográficos dos profissionais desenvolve-se questionário com as variáveis: idade, gênero, escolaridade, situação conjugal, cor, renda familiar, tempo de formação e profissão.

Para a avaliação da qualidade do AACR o Instrumento para Avaliação do Acolhimento com Classificação de Risco. Este instrumento é formado por 21 itens dispostos em escala pontuada, do tipo Likert, de um a cinco (o número 1 corresponde a opção "discordo totalmente" e o número 5, "concordo totalmente"), estruturado de acordo com as três dimensões da avaliação: estrutura, processo e resultado. ${ }^{9}$

Nessa abordagem do instrumento, a Estrutura (itens 1 a 7) se refere aos atributos da instalação onde é prestado o atendimento, incluindo: recursos humanos, materiais, financeiros e estrutura organizacional; o Processo (Itens 8 a 14) tem a ver com as atividades realizadas para a prestação do atendimento e as relações estabelecidas entre os profissionais e usuários; e, por fim, o Resultado (Itens 15 a 21), são os efeitos na saúde e as mudanças de comportamento dos usuários, obtidos a partir da atenção recebida. ${ }^{9}$ 


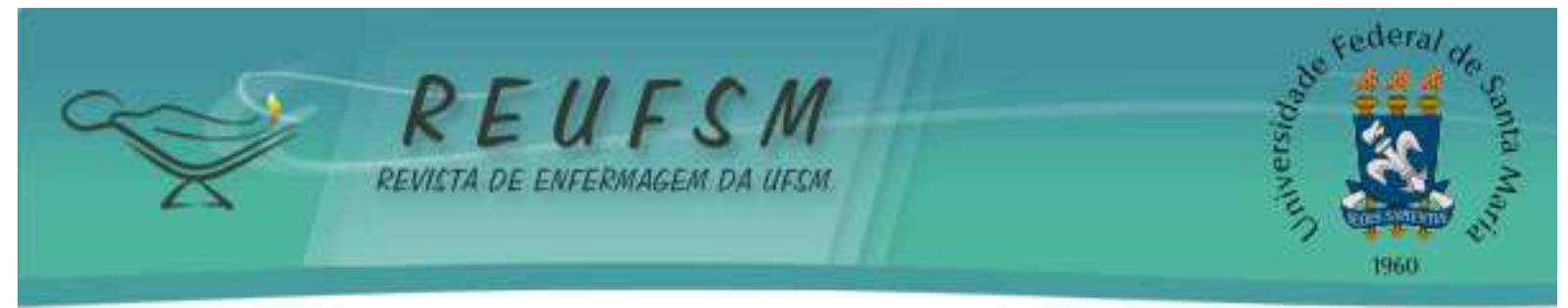

Em relação à avaliação de cada dimensão (estrutura, processo e resultado), a pontuação mínima a ser alcançada é sete e a máxima 35, com amplitude total de 28 pontos, conforme consta no Quadro 1. ${ }^{9}$

\begin{tabular}{|c|c|c|c|}
\hline Pontuação média & Intervalo de classe & Percentual (escores) & Avaliação da Dimensão \\
\hline 31,5 a 35 & 3,5 & 90 a $100 \%$ & Ótimo \\
\hline 26,2 a 31,4 & 5,4 & 75 a $89,9 \%$ & Satisfatório \\
\hline 17,5 a 26,1 & 8,6 & 50 a $74,9 \%$ & Precário \\
\hline 7 a 17,4 & 10,4 & 0 a $49,9 \%$ & Insuficiente \\
\hline
\end{tabular}

Quadro 1 - Escores para pontuação das dimensões de avaliação do ACCR.

Utilizou-se análise descritiva para a caracterização sociodemográfica, profissional e avaliação do AACR. Para as variáveis contínuas calcularam-se, média, desvio padrão, mediana, mínimo e máximo e, para as variáveis categóricas, frequência e percentual.

O presente estudo foi aprovado, no dia 04 de fevereiro de 2016, pelo Comitê de Ética em Pesquisa da Universidade Federal de São Paulo (UNIFESP) (CAAE: 52029815.8.0000.5505).

\section{RESULTADOS}

Foram incluídos 133 profissionais de enfermagem (enfermeiro, técnico e auxiliar de enfermagem) que atuavam no AACR há pelo menos três meses. A média de idade dos participantes foi 36,2 anos $(\mathrm{DP}=8,0)$, sendo a maioria do sexo feminino $(77,4 \%)$, branco $(54,1 \%)$, solteiro $(43,2 \%)$, que exerce a função de técnico/auxiliar de enfermagem $(67,7 \%)$, formado há mais de 5 anos $(59,1 \%)$, de experiência como enfermeiro entre 1 a 3 anos $(33,9 \%)$ e de trabalho na instituição mais de 5 anos (36,8\%). A maioria dos profissionais declarou renda pessoal entre 3 a 5 salários mínimos $(45,9 \%)$ e o número de dependentes é de uma a duas pessoas.

A avaliação da qualidade do AACR do serviço de emergência, de acordo com a percepção dos profissionais de enfermagem mostrou que a média da pontuação das dimensões estrutura, processo e resultado foram, respectivamente, 23,0;22,0 e 20,9, o que indica que os profissionais consideraram precárias as três dimensões avaliadas, como pode ser observado na Tabela 1. 


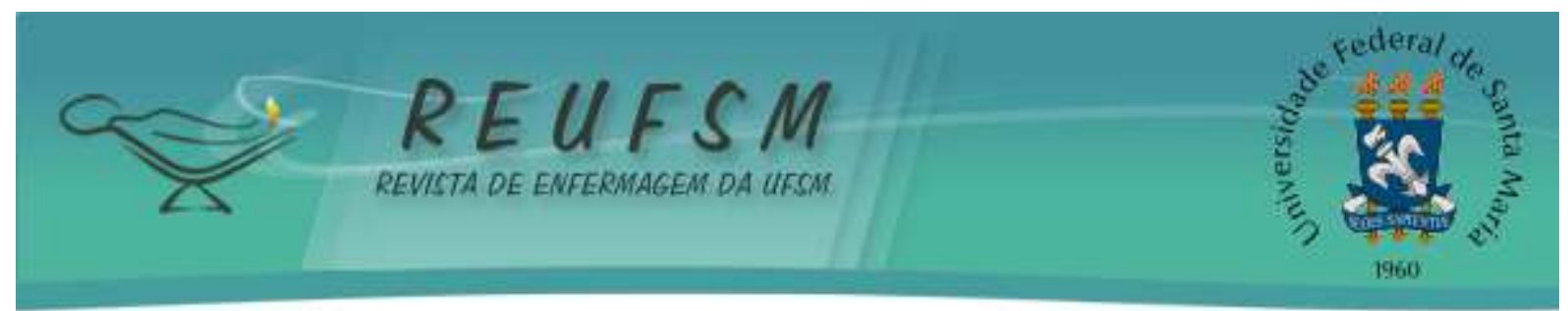

Tabela 1- Avaliação do acolhimento com classificação de risco nas dimensões estrutura, processo e resultado. São Paulo, Brasil, 2016.

\begin{tabular}{l} 
Variáveis \\
\hline Dimensão Estrutura (média da pontuação) \\
A estrutura física para o Acolhimento com Classificação de Risco promove conforto ao \\
usuário e acompanhante. \\
O Acolhimento com Classificação de Risco torna o ambiente mais acolhedor e humano.
\end{tabular}

Escores

(desvio

padrão)

$23,0(3,6)$

O Acolhimento com Classificação de Risco torna o ambiente mais acolhedor e humano.

$2,4(1,1)$

$3,0(1,1)$

Não ocorrem reuniões e treinamentos periódicos para os trabalhadores que atuam no

Acolhimento com Classificação de Risco.

O espaço físico para consulta medica e de enfermagem não proporcionam privacidade ao usuário.

O espaço físico não e suficiente para acolher o acompanhante.

A sinalização do ambiente e clara e suficiente para o direcionamento do usuário ao atendimento no Acolhimento com Classificação de Risco.

Os trabalhadores que atuam no Acolhimento com Classificação de Risco não se comunicam frequentemente com toda equipe do setor.

Dimensão Processo (média da pontuação)

Todos os pacientes não graves que procuram por atendimento nesse serviço de emergência passam pelo Acolhimento com Classificação de Risco.

As condutas a serem tomadas descritas no protocolo de Acolhimento com Classificação e Risco não são conhecidas por todos que trabalham no setor.

As lideranças desse serviço discutem com os funcionários de forma democrática e acolhedora as propostas de mudanças no serviço de Acolhimento com Classificação de Risco.

Os Profissionais que atuam no Acolhimento com Classificação de Risco contribuem para que o usuário se sinta seguro e confortável.

O Fluxograma de atendimento do Acolhimento com Classificação de Risco e discutido com a equipe e avaliado periodicamente quanto à clareza e objetividade.

$\mathrm{O}$ atendimento primário ao usuário ocorre de acordo com a gravidade do caso e não de acordo com a ordem de chegada.

O usuário que não corre risco imediato, assim como a seus familiares, e informado sobre o tempo provável de espera pelo atendimento.

Dimensão Resultado (média da pontuação)

Os profissionais que atuam no Acolhimento com Classificação de Risco estão treinados para atender ao usuário e acompanhante de forma acolhedora e humana.

Nesse serviço, a humanização no atendimento não se faz presente em todas as etapas do atendimento ao usuário.

A equipe multiprofissional que atua nesse serviço trabalha de forma integrada e atenta as necessidades dos usuários.

Os usuários que passam pelo Acolhimento com Classificação de Risco e aguardam pelo atendimento médico não são reavaliados periodicamente.

Mesmo com o Acolhimento com Classificação de Risco os pacientes graves não são priorizados para o atendimento.

Os casos de baixa complexidade não são encaminhados à rede básica de saúde.

Os profissionais que atuam nesse setor se sentem satisfeitos com a implantação do Acolhimento com Classificação de Risco no atendimento. 


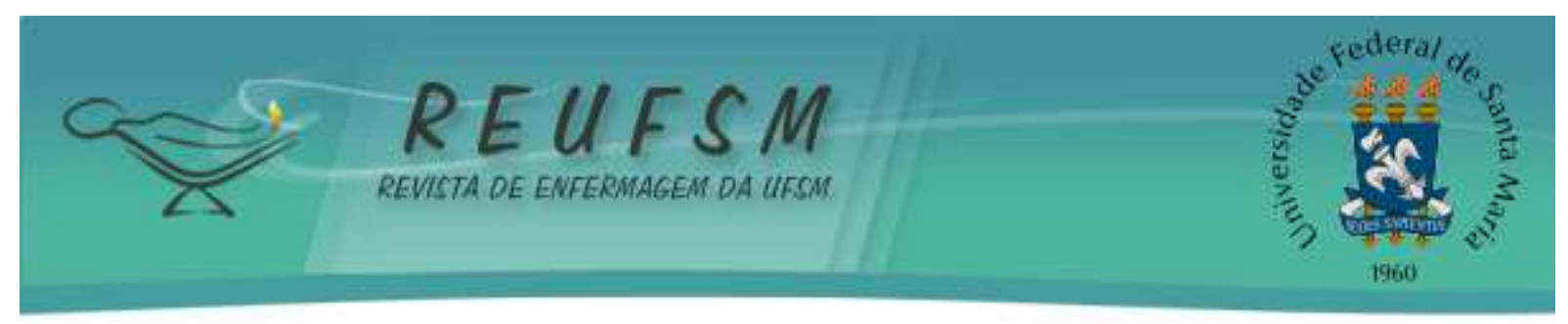

\section{DISCUSSÃO}

Os serviços de emergência têm sido foco de discussões no que se refere à sua organização e integração com outros serviços de saúde. Uma das estratégias adotadas para organizar o atendimento nos SE é o AACR, que tem proporcionado melhorias nos SE, configurando uma ferramenta de apoio à obtenção da qualidade no atendimento dos serviços de emergências dos hospitais brasileiros. ${ }^{2}$

Algumas características da amostra deste estudo como média de idade (36,2 anos) e a maioria ser do sexo feminino $(77,4 \%)$, foram similares as encontradas em outra pesquisa realizada na região Noroeste do estado do Paraná em quatro Serviços Hospitalares de Emergência em que, a média de idade foi 40,5 anos e as mulheres representaram $63,7 \%$ dos entrevistados. ${ }^{10}$ Esta característica confirma os aspectos historicamente relacionados à profissão e à caracterização do perfil de trabalhadores no ambiente hospitalar, em virtude da enfermagem ser uma profissão predominantemente feminina. ${ }^{11}$

Além disso, observou-se que a maior parte dos participantes era de cor branca 54,0\%, uma possível explicação seria a dificuldade de outras etnias ingressarem no ensino superior e profissional, sendo a cor da pele um obstáculo a entrada no mercado de trabalho. ${ }^{12}$ Dos entrevistados, a maioria tinha nível médio de escolaridade (36,6\%), sendo que $67,7 \%$ exerciam função de técnico/auxiliar de enfermagem. Estes achados corroboram com pesquisa realizada em serviços de emergência de dois hospitais beneficentes, sem fins lucrativos, situados no município de Maringá-PR, com atendimento à demanda particular, de operadoras de planos privados de saúde e do Sistema Único de Saúde (SUS) e dois hospitais gerais públicos estaduais, situados na cidade de Londrina-PR, em que a maior parte dos profissionais tinha nível médio de escolaridade $(55,8 \%)$ e a categoria profissional mais frequente foi a de técnico em Enfermagem $(67,3 \%) .{ }^{12}$

Dos enfermeiros 33,9\% relatam ter experiência de 1 a 3 anos. Associado a isto, se observa que $59,1 \%$ dos funcionários tinham formação superior há cinco anos. Estes resultados são semelhantes ao estudo realizado no acolhimento e classificação de risco adulto de um hospital geral e público de Campos dos Goytacazes, Rio de Janeiro, Brasil. ${ }^{13}$ Infere-se a dificuldade de inserção do profissional no mercado de trabalho, que pode estar relacionada a experiência que muitos serviços de saúde exigem, e, também, a própria insegurança do recémformado. Dos participantes desta pesquisa, 36,9\% referiram trabalhar na instituição há mais de 5 anos, o que também foi evidenciado em diversas pesquisas realizadas no Brasil. ${ }^{10-13} \mathrm{O}$ 


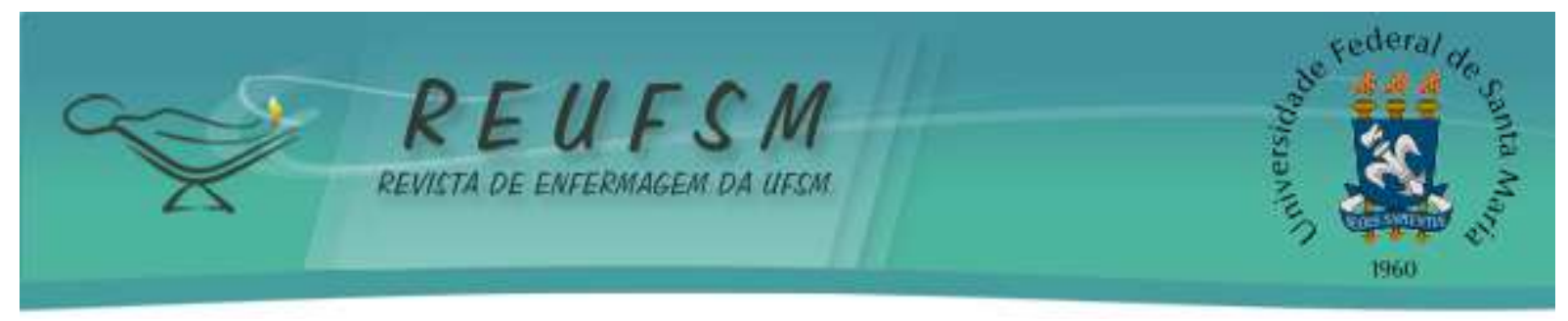

tempo de atuação é importante e deve ser considerado quando propõe-se obter a opinião do sujeito sobre a utilização do AACR. ${ }^{13}$

Em relação à avaliação dos profissionais sobre o AACR destacou-se um parecer precário das dimensões: estrutura $(23,0)$, processo $(22,0)$ e resultado $(20,9)$, o que pode apontar a presença de vulnerabilidades no serviço, As quais deveriam ser examinadas com o intuito de melhorar a assistência prestada. Estes resultados são semelhantes aos de outra pesquisa realizada em serviços de emergência de dois hospitais beneficentes, sem fins lucrativos, situados no município de Maringá-PR, com atendimento à demanda particular, de operadoras de planos privados de saúde e do Sistema Único de Saúde (SUS) e dois hospitais gerais públicos estaduais, situados na cidade de Londrina-PR em que a avaliação do AACR pelos profissionais também foi precária, cujas dimensões receberam as pontuações: estrutura $(23,0)$, processo $(24,0)$ e resultado $(26,0) .{ }^{12}$ A avaliação precária da qualidade dos serviços hospitalares de emergência pode ter relação com a realidade das instituições de saúde brasileiras, muitas vezes caracterizadas por superlotação, estrutura física inadequada, carência de recursos humanos e sobrecarga de trabalho, resultando em avaliações baixas obtidas naquela pesquisa para com os itens: falta de encaminhamento dos casos de baixa complexidade à rede básica de saúde; espaço físico inadequado para os acompanhantes e; falta de discussão/avaliação periódica do fluxo do ACCR. ${ }^{12}$

Neste estudo, o item estrutura obteve uma pontuação de 23,0. O local em que é realizada a entrevista deve ser acolhedor, proporcionando ao usuário e ao seu acompanhante conforto e segurança para relatar o que está acontecendo e qual é o motivo de sua busca pelo serviço. ${ }^{13}$

O processo, com uma pontuação de 22,0, também foi considerado como precário. É importante a utilização de protocolos atualizados e que os funcionários da instituição tenham acesso aos mesmos. Isto pode evitar que ocorram procedimentos desnecessários com os pacientes. ${ }^{14}$ Cabe ressaltar que, mesmo diante do cenário de caos, observado nas portas dos serviços de emergência, causados pela alta demanda de usuários com problemas de saúde/doença, que poderiam ser resolvidos na atenção primária, prestar assistência por meio do ACCR, mesmo em casos não graves, parece ser a conduta mais assertiva para se efetivar o cuidado humanizado nesses serviços. No que tange ao atendimento de usuários não graves por meio do AACR, ressalta-se a importância do conhecimento científico do enfermeiro para 


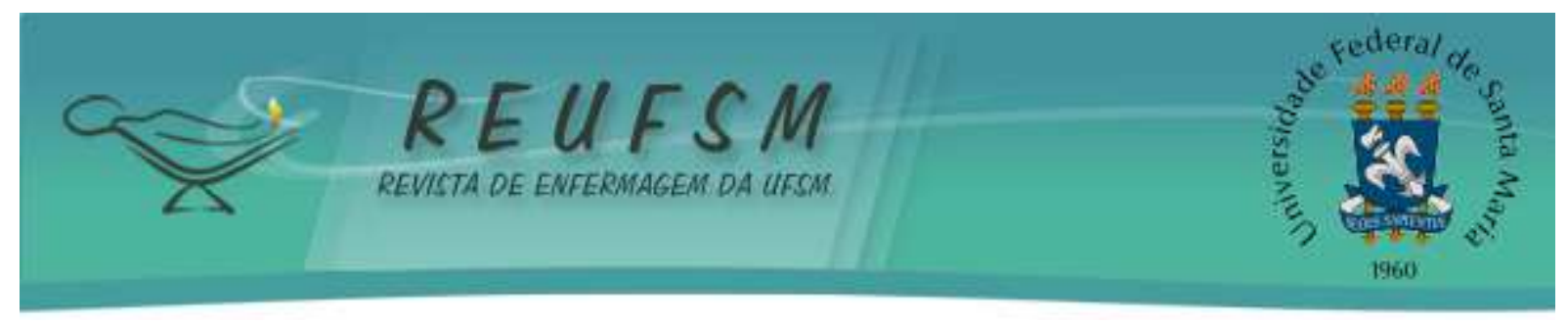

identificar as reais necessidades de saúde nos serviços emergenciais, sem perder o foco na humanização como fator inerente ao processo de cuidado. ${ }^{15}$

A dimensão, apresentou um escore de 20,9 o que a classifica como precária. Achado semelhante foi encontrado em outro estudo realizado no estado do Paraná em dois Serviços Hospitalares de Emergência, em que ambos os serviços, apresentaram resultado como precário. ${ }^{16}$ Esta dimensão talvez seja uma das mais difíceis de ser avaliada, pois encontra-se uma estreita relação entre o atendimento prestado e as constantes alterações no estado de saúde do indivíduo, assim como o seu conhecimento da doença. ${ }^{13} \mathrm{O}$ AACR veio como organizar a assistência de forma mais humanizada e igualitária. Considerando que a dimensão resultado obteve uma avaliação precária, se a necessidade do serviço rever os seus processos e criar métodos que possam ajudar a melhorar o trabalho dos profissionais que ali atuam, o que poderá refletir em um atendimento mais seguro ao usuário. ${ }^{14}$

Os resultados desta pesquisa foram similares a outras realizas com os profissionais dos serviços de emergência, porém em uma em particular, realizada no Paraná observa-se que das três dimensões o resultado obteve maior pontuação. ${ }^{12}$

As limitações deste estudo foram a realização em centro único, a utilização de protocolo desenvolvido localmente e a dificuldade de encontrar literatura sobre a temática, o que limita a comparação com outros estudos e que pode dificultar a generalização dos resultados para outras populações e regiões do país. Os resultados, porém, demonstram a importância da avaliação dos protocolos institucionais pelos profissionais que atuam diretamente no AACR, visando a melhoria dos processos e consequentemente, a uma assistência mais segura ao paciente.

\section{CONCLUSÃO}

$\mathrm{Na}$ avaliação dos profissionais que trabalham no AACR estrutura, processo e resultado foram classificados como precárias. Isto pode refletir a necessidade de reestruturação e monitorização do protocolo institucional. A percepção dos profissionais pode ser mais positiva se os protocolos institucionais forem revistos e se eles forem incluídos nesta reestruturação.

Os apontam que o processo de atendimento do ACCR, no serviço investigado, necessita de melhorias. Espera-se que esta pesquisa promova reflexões e avanços, principalmente no 


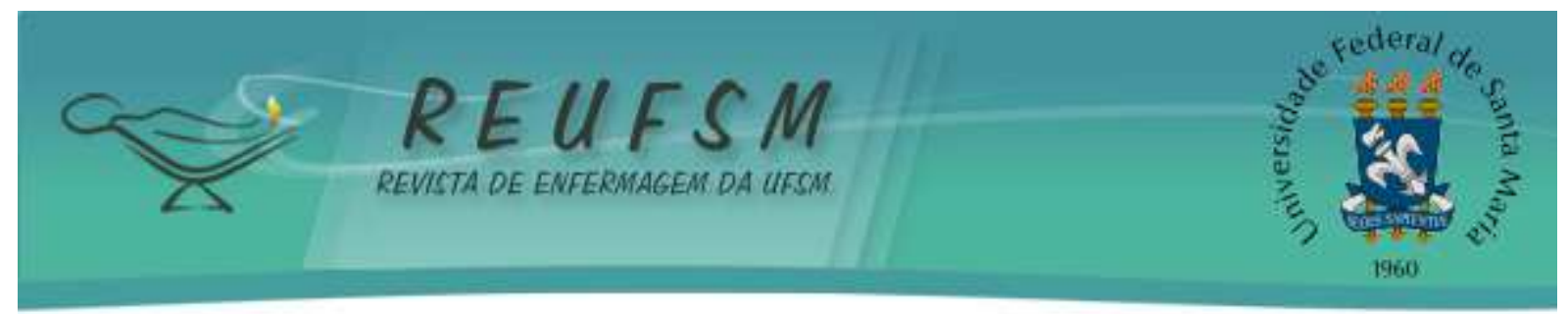

que se refere à atuação do enfermeiro no ACCR, por ser ele, o profissional responsável pela classificação de risco.

O AACR no Brasil é uma modalidade em processo de construção e implantação, a qual faz parte da proposta do Humaniza SUS, portanto, necessita de avaliações contínuas para melhorias. Cabe aqui salientar a escassez de estudos abordando esta temática. A avaliação da qualidade por parte do enfermeiro é de suma importância, pois é ele quem faz a classificação de risco e quando este dispositivo torne-se ineficiente há o comprometimento da assistência prestada aos pacientes.

\section{REFERÊNCIAS}

1. Giunta DH, Pedretti AS, Elizondo CM, Ratti MFG, Quirós FGB, Waisman GD, et al. Descripción de las características del fenómeno Crowding en la Central de Emergencia de Adultos, en un hospital universitario de alta complejidad: estudio de cohorte retrospectiva. Rev Med Chile [Internet]. 2017 [acesso em 2018 jun 06];145:557-63. Disponivel em: https://scielo.conicyt.cl/pdf/rmc/v145n5/art01.pdf.

2. Oliveira GN, Campanharo CRV, Okuno MFP, Batista REA. Acolhimento com avaliação e classificação de risco: concordância entre os enfermeiros e o protocolo institucional. Rev Latinoam Enferm [Internet]. 2013 [acesso em 2018 jun 06];21(2):[07 telas]. Disponível em: http://www.scielo.br/pdf/rlae/v21n2/pt_0104-1169-rlae-21-02-0500.pdf.

3. Vieira AC, Bertoncello KCG, Girondi JB, Nascimento ERP, Hammerschmidt KSA, Zefeino MT. Percepção de enfermeiros de emergência no uso de protocolo de avaliação de dor torácica. Texto \& Contexto Enferm [Internet]. 2016 [acesso em 2018 jun 06];25(1):e1830014. Disponível em: http://www.scielo.br/pdf/tce/v25n1/pt_0104-0707-tce25-01-1830014.pdf.

4. Fradique MJ, Mendes L. Efeitos da liderança na melhoria da qualidade do cuidado de enfermagem. Rev Enf Ref [Internet]. 2013 [acesso em 2018 jun 06];III(10):45-53. Disponível em: http://www.scielo.mec.pt/scielo.php?script=sci_arttext\&pid=S087402832013000200006 .

5. Inoue KC, Bellucci Júnior JA, Papa MA, Vidor RC, Matsuda LM. Avaliação da qualidade da classificação de risco nos serviços de emergência. Acta Paul Enferm [Internet]. 2015 [acesso em 2017 set 06];28(5):420-25. Disponível em: http://www.scielo.br/pdf/ape/v28n5/1982-0194-ape-28-05-0420.pdf.

6. Anschau F, Webster J, Roessler N, Fernandes EO, Klafke V, Silva CP, et al. Avaliação de intervenções de gestão da clínica na qualificação do cuidado e na oferta de leitos em um hospital público de grande porte. Sci Med [Internet]. 2017 [acesso em 2017 set 06];27(2):1-7. Disponível em: http://dx.doi.org/10.15448/1980-6108.2017.2.26575.

7. Brasil. Ministério da Saúde. Secretaria-Executiva. Núcleo Técnico da Política Nacional de Humanização. HumanizaSUS: acolhimento com avaliação e classificação de risco: um paradigma ético-estético no fazer em saúde. Brasília (DF): Editora do Ministério da Saúde; 2004. p. 49. (Série B. Textos Básicos de Saúde). 


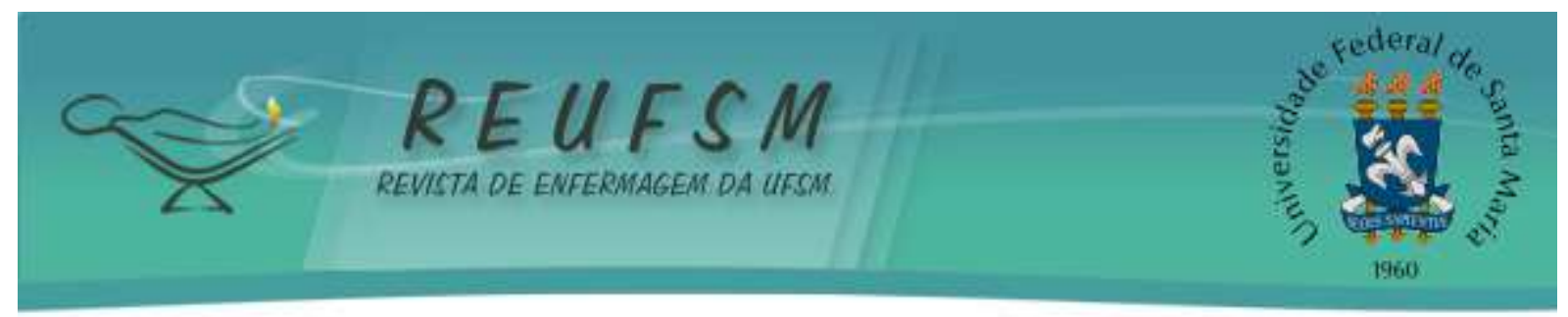

8. Brasil. Ministério da Saúde. Secretaria de Atenção à Saúde. Política Nacional de Humanização da Atenção e Gestão do SUS. Humaniza SUS - Acolhimento e Classificação de Risco nos Serviços de Urgência. $1^{a}$ ed. Brasília, DF: Editora do Ministério da Saúde; 2009. p. 60 (Série B. Textos Básicos de Saúde).

9. Souza GJ, Paula MAB, Salles PJ. O perfil sócio demográfico de trabalho da equipe de enfermagem que atua na sala de emergência de uma unidade mista. Rev Rede Cuidados Saúde [Internet]. 2015 [acesso em 2017 set 06];9(3):1-17. Disponível em: http://publicacoes.unigranrio.edu.br/index.php/rcs/article/view/2728/1504.

10. Bellucci Júnior JA, Vituri DW, Versa GLGS, Furuya PS, Vidor RC, Matsuda LM. Acolhimento com classificação de risco em serviço hospitalar de emergência: avaliação do processo de atendimento. Rev Enferm UERJ [Internet]. 2015 [acesso em 2017 set 06];23(1):82-7. Disponível em: http://dx.doi.org/10.12957/reuerj.2015.4976.

11. Souza GJ, Paula MAB, Salles PJ. O Perfil sócio demográfico de trabalho da equipe de enfermagem que atua na sala de emergência de uma unidade mista. Rev Rede Cuidados Saúde [Internet]. 2015 [acesso em 2017 set 06];9(3):1-17. Disponível em: http://publicacoes.unigranrio.edu.br/index.php/rcs/article/view/2728/1504.

12. Versa GLGS, Vituri DW, Buriola AA, Oliveira CA, Matsuda LM. Avaliação do acolhimento com classificação de risco em serviços de emergência hospitalar. Rev Gaúcha Enferm. 2014;35(3):21-8.

13. Araújo YB, Ferreira LBA, Santos CM, Silva ATMF, Gomes MSM. Acolhimento e classificação de risco nos serviços de urgência e emergência: limites e possibilidades uma questão para enfermeiro. Persp Online: Biol \& Saúde [Internet]. 2014 [acesso em 2017 set 06];15(4):25-49. Disponível em: http://www.seer.perspectivasonline.com.br/index.php/biologicas_e_saude/article/view/566/489.

14. Versa GLGS, Vituri DW, Bellucci Junior JA, Zampieri ACP, Alves SR, Matsuda LM. Acolhimento com classificação de risco: avaliação da dimensão resultado na percepção de trabalhadores. Cienc Cuid Saude. 2016;15(1):85-92.

15. Inoue KC, Bellucci Júnior JA, Papa MA, Vidor RC, Matsuda LM. Avaliação da qualidade da classificação de risco nos serviços de emergência. Acta Paul Enferm [Internet]. 2015 [acesso em 2018 jun 06];28(5):420-5. Disponível em: http://www.scielo.br/pdf/ape/v28n5/1982-0194-ape-28-05-0420.pdf.

16. Costa MAR, Versa GLGS, Bellucci Júnior JA, Inoue KC, Sales CA, Matsuda LM. Acolhimento com classificação de risco: avaliação de serviços hospitalares de emergência. Esc Anna Nery [Internet]. 2015 [acesso em 2018 jun 06];19(3):491-7. Disponível em: http://www.scielo.br/scielo.php?script=sci_arttext\&pid=S1414-81452015000300491.

Data de submissão: 22/08/2017

Data de aceite: 04/07/2018

Autor correspondente: Maria Carolina Barbosa Teixeira Lopes

E-mail: lopes.carolina@unifesp.br

Endereço: Rua Napoleão de Barros, 754 - Vila Clementino, São Paulo - SP

CEP: 04024-002 